\title{
Adaptive Channel Estimation Using Cyclic Prefix in Multicarrier Modulation System
}

\author{
Xiaowen Wang and K. J. Ray Liu
}

\begin{abstract}
Multicarrier modulation (MCM) is a promising technique for high rate data transmission and the channel estimation is very important for the implementation of MCM. We found that the cyclic prefix, originally used solely to reduce the intersymbol interference (ISI), can be viewed as a source of channel information. Based on this observation, we propose in this paper an adaptive channel estimation algorithm using the cyclic prefix. This algorithm can adaptively track the channel variation without additional training sequences.
\end{abstract}

Index Terms - Channel estimation, cyclic prefix, multicarrier modulation.

\section{INTRODUCTION}

$\mathbf{M}$ ULTICARRIER modulation (MCM) is now considered an effective technique for high rate data communications in both wire and wireless environments [1], [2]. MCM partitions the channel bandwidth into several parallel independent subchannels. It has a relatively longer symbol duration which produces greater immunity to impulse noise and fast fading, and moreover, the independence of the subchannels provides a simple equalization scheme and a natural way to optimize the channel capacity usage.

Correct channel estimation is very important to the implementation of the MCM system. Although some techniques, such as differential PSK modulation, can be used to eliminate the need for channel estimation, they cause some performance loss. In most systems, a known training sequence is sent by the transmitter and a training algorithm is performed by the receiver on the observed channel output and the known input to estimate the channel [3], [4]. The deterministic least squares (DLS) channel identification algorithm in [4] is such a simple but widely used training approach. However, it is not suited for time varying system. In practice, we either assume that the channel is invariant and use the initial training to get the channel estimation [3], or periodically employ training sequences to track the channel variation [4]. These two solutions will either cause performance loss or increase the overhead of the system.

In this letter, we present that the cyclic prefix in MCM system which is used to reduce intersymbol interference (ISI) and normally discarded at the receiver can be viewed as a constantly sent training sequence for channel estimation. Hence we can use it to adaptively estimate the channel without additional training sequences.

Manuscript received December 23, 1998. The associate editor coordinating the review of this letter and approving it for publication was Prof. Y. BarNess.

The authors are with the Electrical Engineering Department, University of Maryland, College Park, MD 20742 USA.

Publisher Item Identifier S 1089-7798(99)08042-4.

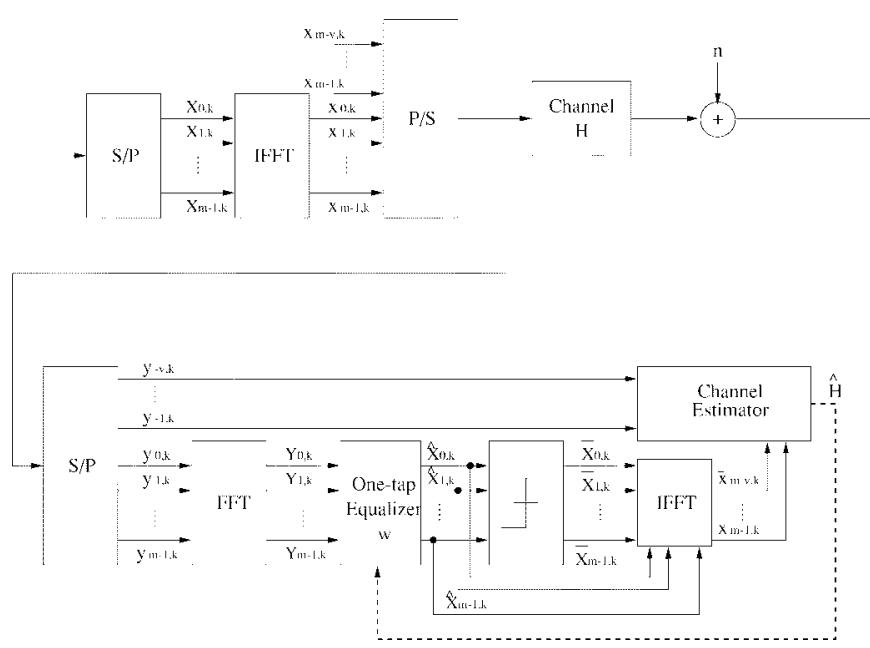

Fig. 1. The proposed MCM system with cyclic prefix and adaptive channel estimation.

\section{The Proposed Adaptive Channel Estimation ALGORITHM}

\section{A. MCM System Using Cyclic Prefix}

Fig. 1 shows a MCM system using cyclic prefix. The system has $m / 2$ complex parallel subchannels. Input data are first buffered to blocks and then divided into $m / 2$ bit streams and mapped to some complex constellation points $X_{i, k}, i=$ $1, \cdots, m / 2$ at time $k$. The modulation is implemented by performing $m$-point inverse discrete Fourier transform (IDFT) on $\mathbf{X}_{k}=\left[X_{0, k} X_{1, k} \cdots X_{m-1, k}\right]^{T}$. The last $m / 2$ samples of $\mathbf{X}_{k}$ are just the conjugate of the first $m / 2$ samples so that the output of IDFT, $\mathbf{x}_{k}=\left[x_{0, k} x_{1, k} \cdots x_{m-1, k}\right]^{T}$ consists of all real samples.

The channel is usually modeled as a FIR filter with length $v$. The impulse response of the channel is $\mathbf{h}=\left[h_{0}, h_{1}, \cdots, h_{v}\right]^{T}$. A cyclic prefix $\mathbf{x}_{k}^{(f)}=$ $\left[x_{-v, k} \cdots x_{-1, k}\right]^{T}$, where $x_{-i, k}=x_{m-i, k}, i=1, \cdots, v$, is appended in front of $\mathrm{x}_{k}$ before transmission. At the receiver, the prefix part $\mathbf{y}_{k}^{(f)}=\left[y_{-v, k} \cdots y_{-1, k}\right]^{T}$ is discarded. The demodulation is performed only on $\mathbf{y}_{k}=\left[y_{0, k} y_{1, k} \cdots y_{m-1, k}\right]^{T}$ by the DFT operation and the demodulated signal is $\mathbf{Y}_{k}=\left[Y_{0, k} Y_{1, k} \cdots Y_{m-1, k}\right]^{T}$.

The cyclic prefix reduces the ISI between $\mathrm{X}_{k}$ 's and makes the subchannels independent with each other. Therefore, for each subchannel, we have

$$
Y_{i, k}=X_{i, k} \mathcal{H}_{i}+N_{i, k}
$$

where $\mathcal{H}_{i}=\sum_{l=0}^{v} h_{l} e^{-j 2 \pi i l / m}$ is the channel frequency response and $N_{i, k} \sim \mathcal{N}\left(0, \sigma^{2}\right)$ is the noise of the $i$ th subchannel. 


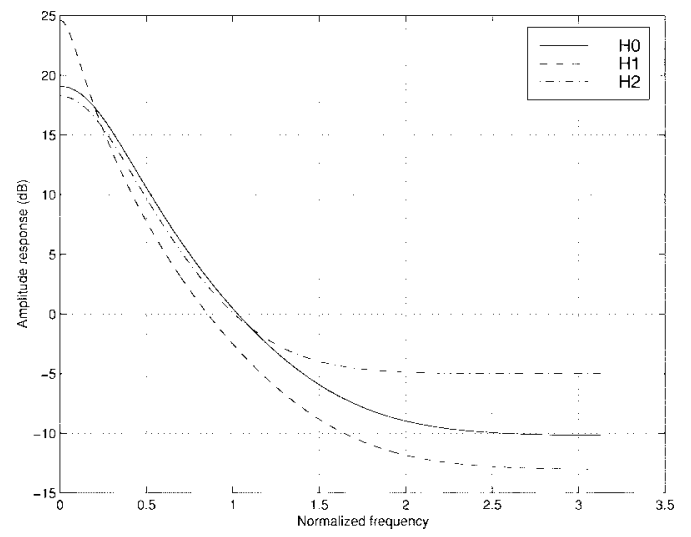

(a)

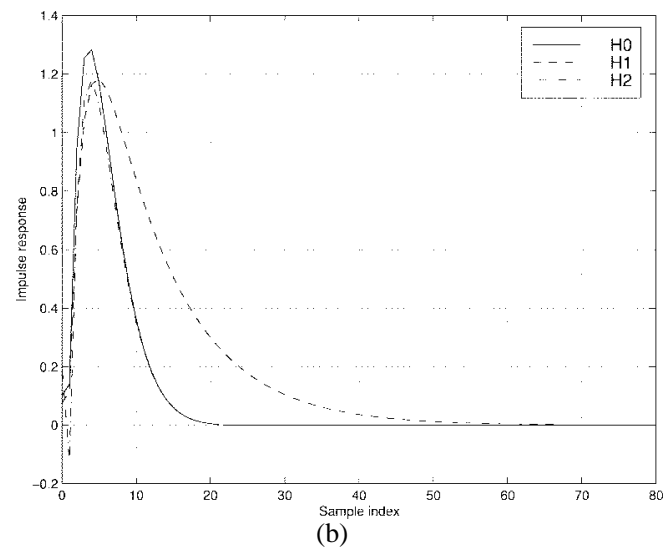

Fig. 2. Channel responses: (a) frequency response and (b) impulse response.

According to (1) only a one-tap equalizer $w_{i}$ is needed to get the optimal MMSE estimation of $X_{i, k}$, i.e., $\hat{X}_{i, k}=Y_{i, k} \cdot w_{i}$. The coefficient of the one-tap equalizer for $i$ th subchannel is

$$
w_{i}=\frac{\Gamma_{i}^{1 / 2} \mathcal{H}_{i}^{*}}{\Gamma_{i}\left\|\mathcal{H}_{i}\right\|^{2}+\sigma_{i}^{2}}, \quad i=0, \cdots, m-1
$$

where $\Gamma_{i}=E\left[\left\|X_{i, k}\right\|^{2}\right]$. The decision is then made on $\hat{X}_{i, k}$ to get the final output $\bar{X}_{i, k}=q\left(\hat{X}_{i, k}\right), i=0, \cdots, m-1$, where $q(\cdot)$ is the decision operation.

\section{B. Observation on Cyclic Prefix}

The channel information in (2) is usually obtained by training process. For a time invariant channel, only initial training is needed. However, for a time varying channel, retraining must be done periodically to keep on with the channel variations, otherwise, the system performance degrades. Obviously, such a scheme increases the overhead of the system. In this section we see that by using cyclic prefix, retraining is not necessary in the MCM system to track the channel variations.

Let's first consider the prefix part $\mathbf{y}_{k}^{(f)}$ which is originally discarded. The relationship between $\mathbf{y}_{k}^{(f)}$ and the transmitted signal is

$\mathbf{y}_{k}^{(f)}=\mathbf{A}_{k} \mathbf{h}+\mathbf{n}_{k}^{(f)}$
where $\mathbf{A}_{k}=\left[\begin{array}{cccc}x_{-v, k} & x_{m-1, k-1} & \cdots & x_{m-v, k-1} \\ \ddots & \ddots & & \vdots \\ & \ddots & \ddots & \vdots \\ x_{-1, k} & \cdots & x_{-v, k} & x_{m-1, k-1}\end{array}\right]$ and
$\mathbf{n}_{k}^{(f)}=\left[n_{-v, k} \cdots n_{-1, k}\right]^{T}$. (a)
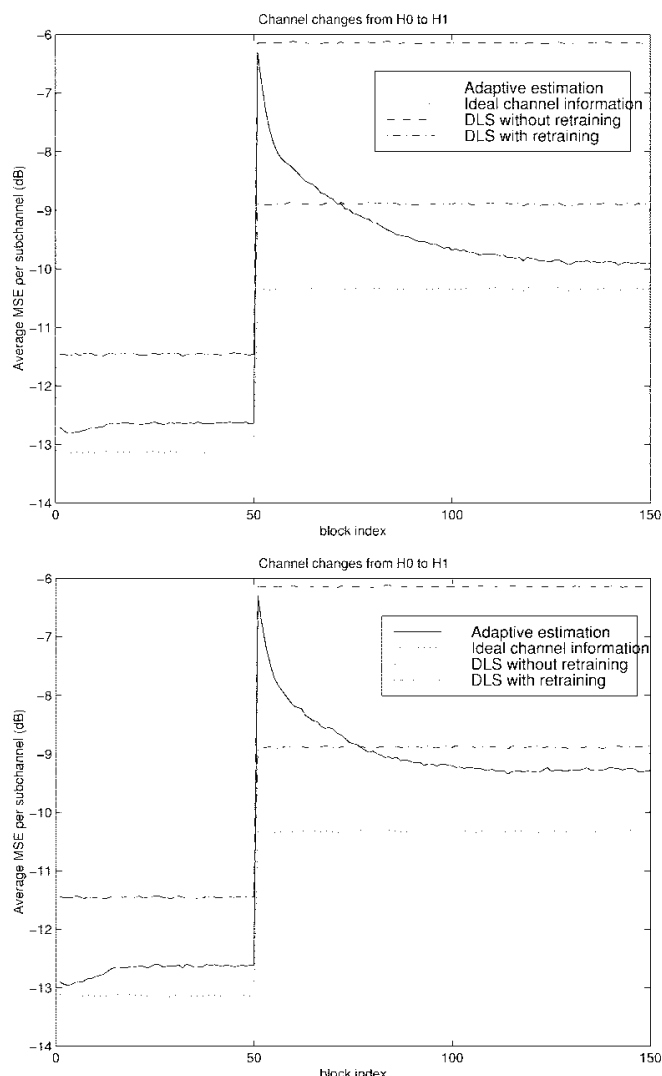

(b)

(c)
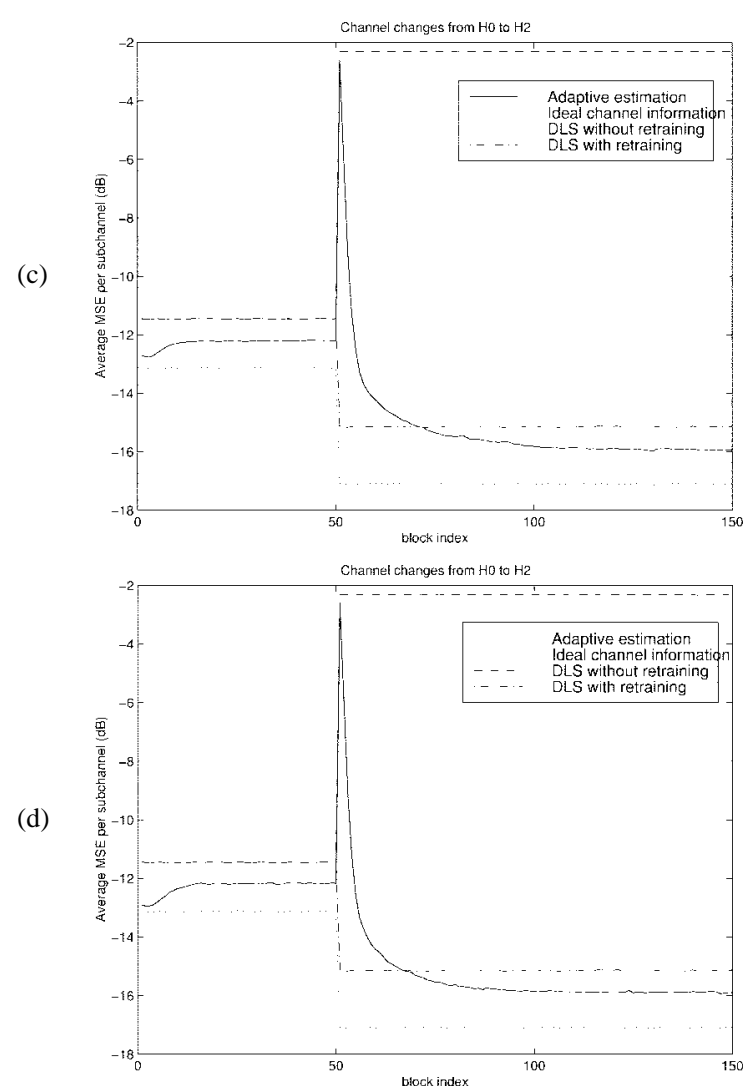

Fig. 3. Average MSE per subchannel $\left(\sigma^{2}=0.01, P e=10^{-7}\right)$. (a) $v=64, \lambda_{1}=0.75, \lambda_{2}=1$. (b) $v=32, \lambda_{1}=0.75, \lambda_{2}=1$. (c) $v=64, \lambda_{1}=0.6, \lambda_{2}=1$. (d) $v=32, \lambda_{1}=0.6, \lambda_{2}=1$.

The lower triangle part of matrix $\mathbf{A}_{k}$ is composed by $\mathbf{x}_{k}^{(f)}$, while the upper triangle part is composed by 
the last $v-1$ samples of $\mathrm{x}_{k-1}$. However, the last $v-1$ samples of $\mathrm{x}_{k-1}$ are also the elements of the prefix $\mathbf{x}_{k-1}^{(f)}$. Hence, if all the prefix parts concatenate together to form a pair of sequences $\left\{x_{l}^{(f)}\right\}=$ $\left\{\cdots x_{-v, k-1} \cdots x_{-1, k-1} x_{-v, k} \cdots x_{-1, k} \cdots\right\} \quad$ and $\left\{y_{l}^{(f)}\right\}=\left\{\cdots y_{-v, k-1} \cdots y_{-1, k-1} y_{-v, k} \cdots y_{-1, k} \cdots\right\}$, the relationship between these two satisfies

$$
y_{l}^{(f)}=x_{l}^{(f)} * h_{l}+n_{l} \text {. }
$$

Equation (4) shows that if we send $\left\{x_{l}^{(f)}\right\}$ to the channel as the training sequence, the channel output is exactly $\left\{y_{l}^{(f)}\right\}$. We can use this training sequence provided by the cyclic prefix to estimate the channel if we can recover $\left\{x_{l}^{(f)}\right\}$ correctly. In our algorithm, the estimations of the transmitted prefix are obtained by performing IDFT on the decision-directed samples $\bar{X}_{i, k}$ 's to reduce the effect of channel noise.

The recursive least-square (RLS) algorithm is chosen to get the channel estimation from (4) because of its good tracking property [6]. Noticing that data in (3) arrive block by block, we propose a block RLS method which updates the channel estimation by blocks. The channel estimation at time $k$ is $\hat{\mathbf{h}}(k)=\boldsymbol{\Phi}^{-1}(k) \mathbf{z}(k)$, where $\boldsymbol{\Phi}(k)=$ $\lambda_{1} \boldsymbol{\Phi}(k-1)+\sum_{l=1}^{v} \lambda_{2} \mathbf{u}_{l}(k) \mathbf{u}_{l}^{H}(k)$ is the approximation of the correlation matrix of the training input $\left\{x_{l}^{(f)}\right\}$ and $\mathbf{z}(k)=\lambda_{1} \mathbf{z}(k-1)+\sum_{l=1}^{v} \lambda_{2} \mathbf{u}_{l}(k) y_{-l, k}^{*}$, is the approximation of the cross-correlation vector between the training input $\left\{x_{l}^{(f)}\right\}$ and output $\left\{y_{l}^{(f)}\right\}$. The data vector $\mathbf{u}_{l}(k)$ is formed as $\mathbf{u}_{l}(k)=\left[x_{-l, k} \cdots x_{-1, k} x_{-v, k-1} \cdots x_{-v+l, k-1}\right]^{T}$, $l=v, v-1, \cdots, 1$. $\lambda_{1}$ and $\lambda_{2}$ are forgetting factors for the data between blocks and within same block, respectively. Usually we choose $\lambda_{2}=1$ and $\lambda_{1}<1$.

\section{Joint Adaptive Channel Estimation and Equalization Algorithm}

Based on the above discussion, we summarize the channel estimation and equalization algorithm as follows:

Input: $\mathbf{y}_{k}^{(f)}$ and $\mathbf{Y}_{k}$.

Known parameters: $\Gamma_{i}$ and $\sigma_{i}^{2}$.

Selecting parameters: $\lambda_{1}$ and $\lambda_{2}$.

Initialization: $k=0$, an initial training process is used to initialize $\hat{\mathbf{h}}(0)$ and $\overline{\boldsymbol{\Phi}}(0)$.

Computation: $k=1,2,3, \cdots$

1) $\mathcal{H}_{i}(k-1)=(1 / \sqrt{m}) \sum_{l=0}^{v} \hat{h}_{l}(k-1) e^{-j 2 \pi i l / m}$, $w_{i}(k-1)=\Gamma_{i}^{1 / 2} \mathcal{H}_{i}^{*}(k-1) / \Gamma_{i}\left\|\mathcal{H}_{i}(k-1)\right\|^{2}+\sigma_{i}^{2}, i=$ $0, \cdots, m-1$

2) $\hat{X}_{i, k}=Y_{i, k} w_{i}(k-1), i=0, \cdots m-1$

3) $\bar{x}_{i, k}=(1 / \sqrt{m}) \sum_{l=0}^{m-1} q\left(\hat{X}_{l, k}\right) e^{j(2 \pi / i l) m}, i=m-$ $v, \cdots, m-1$

4) $\overline{\boldsymbol{\Phi}}(k)=\lambda_{1} \overline{\boldsymbol{\Phi}}(k-1)+\sum_{l=1}^{v} \lambda_{2} \overline{\mathbf{u}}_{l}(k) \overline{\mathbf{u}}_{l}^{H}(k), \overline{\mathbf{z}}(k)=$ $\lambda_{1} \overline{\mathbf{z}}(k-1)+\sum_{l=1}^{v} \lambda_{2} \overline{\mathbf{u}}_{l}(k) y_{-l, k}^{*}$, where $\overline{\mathbf{u}}_{l}(k)=$ $\left[\bar{x}_{-l, k} \cdots \bar{x}_{-1, k} \bar{x}_{-v, k-1} \cdots \bar{x}_{-v+l, k-1}\right]^{T}, \bar{x}_{-l, k}=$ $\bar{x}_{m-l, k}, l=v, v-1, \cdots, 1$

5) $\hat{\mathbf{h}}(k)=\overline{\boldsymbol{\Phi}}^{-1}(k) \overline{\mathbf{z}}(k)$.

Here steps 4 and 5 can be replaced by many existing fast RLS algorithms [6].

\section{Simulation Results}

We use an MCM system with DFT length $m=512$ and QAM constellation in our simulation. The transmit power of all the used subchannels is equal with bit allocation keeping the subchannels with the same preset error probability $P_{e}$. White noise is used, i.e., $\sigma_{i}^{2}=\sigma^{2}$. The performance is evaluated by the averaged mean square error (MSE) per subchannel which is defined as err $=\sum_{i \in U}\left\|X_{i}-\hat{X}_{i}\right\|^{2} /|U|$, where $U$ is the set of all the used subchannels. $|U|$ is the number of all the used subchannels.

The simulation is carried under the digital subscriber line environment. Fig. 2 shows the channel responses used in the simulation. They all have same average squared gain and are generated from ARMA models with same order but different poles or zeros. At the beginning of the simulation, the channel is $H_{0}$. The bit allocation is done according to $H_{0}$ and remains unchanged during the simulation. After some time, the channel changes to $H_{1}$ or $H_{2}$. In our algorithm, only the first block is sent as pure training sequence to get the information about $H_{0}$. Then the real data are sent and the adaptive channel estimation algorithm is used to track the variation of the channel. Fig. 3 shows the results of the simulation. The results of using DLS training algorithm in [4] with and without retraining are also plotted for comparison. It should be noticed that additional training sequences are used which are not represented in the figure in DLS for both initial training and retraining. Fig. 3(a) and (b) shows results with different prefix length when the channel changes from $H_{0}$ to $H_{1}$, while Fig. 3(c) and (d) shows results when the channel changes from $H_{0}$ to $H_{2}$. We can see that the algorithm can adaptively track these variations in about 50 symbols. It has about a $4-\mathrm{dB}$ gain over the system just using initial training and is slightly better than the system using retraining when channel changes, though the tracking is done by our algorithm without additional training.

\section{CONCLUSION}

We have presented an adaptive channel estimation algorithm using the cyclic prefix in MCM systems. This algorithm can adaptively track variation of a moderately time-varying channel, which makes the MCM system more robust to channel variation. Moreover, the algorithm achieves this tracking property in an efficient way without adding any more overhead to the system since no additional training is needed.

\section{REFERENCES}

[1] J. A. C. Bingham, "Multicarrier modulation for data transmission: An idea whose time has come," IEEE Commun. Mag., pp. 5-14, May 1990.

[2] J. M. Cioffi, A Multicarrier Primer.

[3] J. S. Chow, J. C. Tu, and J. M. Cioffi, "A discrete multitone transceiver system for HDSL application," IEEE J. Select. Areas Commun., vol. 9, pp. 895-908, Aug. 1991.

[4] R. A. Ziegler and J. M. Cioffi, "Estimation of time-varying digital radio channel," IEEE Trans. Veh. Technol., vol. 41, pp. 134-151, May 1992.

[5] Y. Li, L. J. Cimini, Jr., and N. R. Sollenberger, "Robust channel estimation for OFDM systems with rapid dispersive fading channels," IEEE Trans. Commun., vol. 46, pp. 902-915, July 1998.

[6] S. Haykin, Adaptive Filter Theory. Englewood Cliffs, NJ: Prentice Hall, 1996. 